\title{
Optical and optomechanical design of the MERLIN laser optical bench
}

M. Livrozet, B. Gronloh, H. Faidel, J. Luttmann, D. Hoffmann

M. Livrozet, B. Gronloh, H. Faidel, J. Luttmann, D. Hoffmann, "Optical and optomechanical design of the MERLIN laser optical bench," Proc. SPIE 11852, International Conference on Space Optics - ICSO 2020, 118522Q (11 June 2021); doi: 10.1117/12.2599445

SPIE Event: International Conference on Space Optics - ICSO 2021, 2021, Online Only 


\section{International Conference on Space Optics-ICSO 2020}

Virtual Conference

30 March-2 April 2021

Edited by Bruno Cugny, Zoran Sodnik, and Nikos Karafolas
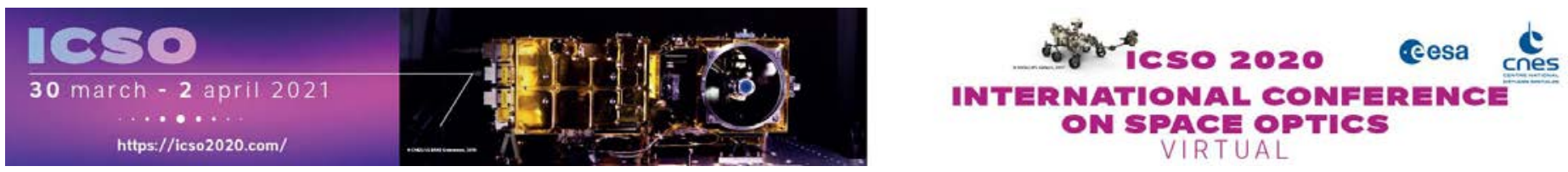

\section{Optical and optomechanical design of the MERLIN laser optical bench}

\section{Cesa isoporeseatings denes}




\title{
Optical and Optomechanical Design of the MERLIN Laser Optical Bench
}

\author{
M. Livrozet ${ }^{\mathrm{a}}$, B. Gronloh ${ }^{\mathrm{a}}$, H. Faidel ${ }^{\mathrm{a}}$, J. Luttmann ${ }^{\mathrm{a}}$, D. Hoffmann ${ }^{\mathrm{a}}$, \\ ${ }^{a}$ Fraunhofer Institute for Laser Technology, Steinbachstr. 15, 52074 Aachen, Germany
}

\begin{abstract}
For the satellite-based methane lidar instrument MERLIN a reliable laser source is needed that emits laser pulses at two wavelengths of around $1645 \mathrm{~nm}$ to measure the methane concentration of earth's atmosphere with an Integrated Path Differential Absorption LIDAR (IPDA). To generate those pulses, the laser (LASO) consists of a seeded, actively qswitched, diode pumped Nd:YAG master oscillator power amplifier (MOPA) and a subsequent seeded and frequencycontrolled optical parametric oscillator (OPO).

Due to the passive thermal control of the instrument the laser has to withstand a large non-operational and operational temperature range and also high mechanical loads while at the same time a compact envelope is required. Together with the demanding requirements on the laser performance a very robust optical design is needed.

To meet those requirements, Fraunhofer Institute for Laser Technology (ILT) uses optomechanical mounts that were developed in a previous project and show very low tilting over a large operational temperature range, even after nonoperational temperature cycling and applying mechanical loads. The mounts are soldered and free of organic substances as the LASO is enclosed in a pressurized housing (LASH). Any outgassing could lead to a decay or damage of the optics and thus a failure of the laser.

During the development of the optomechanical mounts many tests were performed to quantify the statistical behavior under mechanical and thermal loads. Based on those results and additional mechanical simulations, Monte-Carlo-Analyses have been performed to analyze the performance of the laser and to verify the fulfilment of the requirements.
\end{abstract}

Keywords: MERLIN instrument, optical design, LIDAR, spaceborne Laser, Nd:YAG Laser, OPO, single-frequency, soldered optics

\section{INTRODUCTION}

The Methane Remote Sensing LIDAR Mission (MERLIN) is a cooperation between the French Space Agency CNES and the German Space Administration DLR. With the Integrated Path Differential Absorption LIDAR (IPDA) of the instrument the methane concentration and the temporal and local gradients in the earth's atmosphere shall be measured during the mission duration of 3.25 years. An overview of the mission and the instrument design can be found in [1][2] and [3].

The MERLIN LIDAR employs a Nd:YAG laser with a following optical parametric oscillator (OPO) as light source. The laser emits double pulses with two wavelengths around $1645 \mathrm{~nm}$ : The wavelength of one of these pulses is at a broadened absorption feature of methane (lambda_on $=1645.5516 \mathrm{~nm}$ ) while the other wavelength is close to a local minimum of atmospheric absorption (lambda_off $=\sim 1645.825 \mathrm{~nm}$ ). With the known absorption coefficients for both wavelengths, the absolute integrated methane concentration can be derived from the difference between the backscattered signals. The main advantage of this setup is that with the laser as active light source no external illumination is required so that it can operate during night and day and even through thin cloud layers.

In this paper, we present the optical and optomechanical design of the laser optical bench (LASO) as well as tests and analyses that were conducted on the performance. Those results were part of the Critical Design Review (CDR), which was successfully completed in 09/2020.

*marie.livrozet@ilt.fraunhofer.de; phone+49 2418906 8010; ilt.fraunhofer.de

Proc. of SPIE Vol. 11852 118522Q-2 


\section{LASO CONCEPT}

\subsection{System Overview}

The laser optical bench will be mounted in a pressurized laser housing (LASH) with three isostatic mounts at the interfaces. This housing will be provided by the instrument prime Airbus Defence and Space GmbH. Within the housing the pressure will be around 1000 mbar and dry air will be used as gas. The design of the housing was successfully tested on the FULAS laser [4].

The optical bench consists of a baseplate with optical elements on both sides (see Figure 1). The LASO itself does not include any electronics or active controls, but an electrical harness is required to deliver the different electrical currents, voltages and signals. Those inputs are provided by the Laser Electronics (LAE). A dedicated thermal harness is used for direct heat transfer from heat sources inside the laser to the instrument's cooling system. In absence of an active thermal control for the laser baseplate, the thermal harness is thermally decoupled from any structural element of the laser.

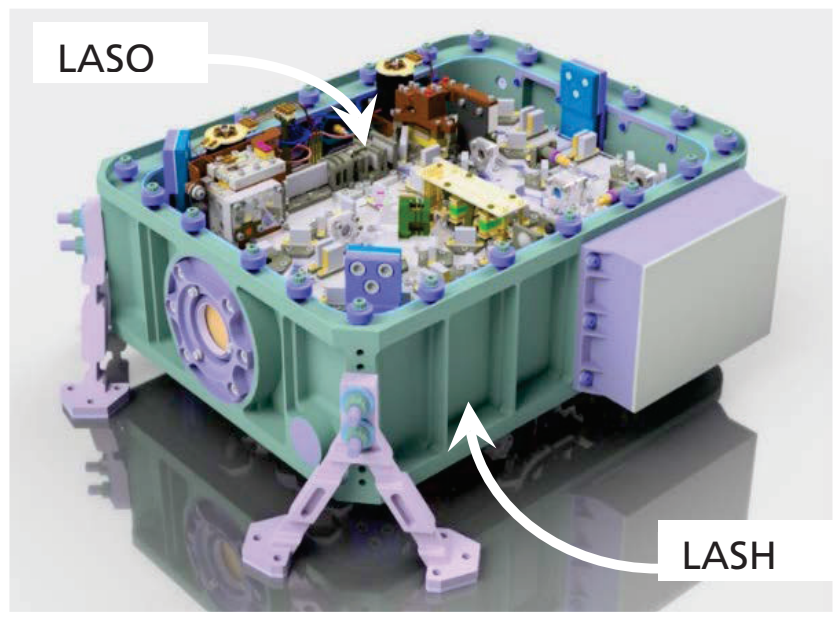

Figure 1. Model of LASO and LASH

To monitor the optical pulse energies at different stages within the laser and deliver the optical signals, that are used for the control of the laser, fibers are used. Those fibers are connected to the Frequency reference unit (FRU), which also delivers the seed signals for the laser and the OPO. The schematic interfaces of the three modules LASO, LAE and FRU are shown in Figure 2.

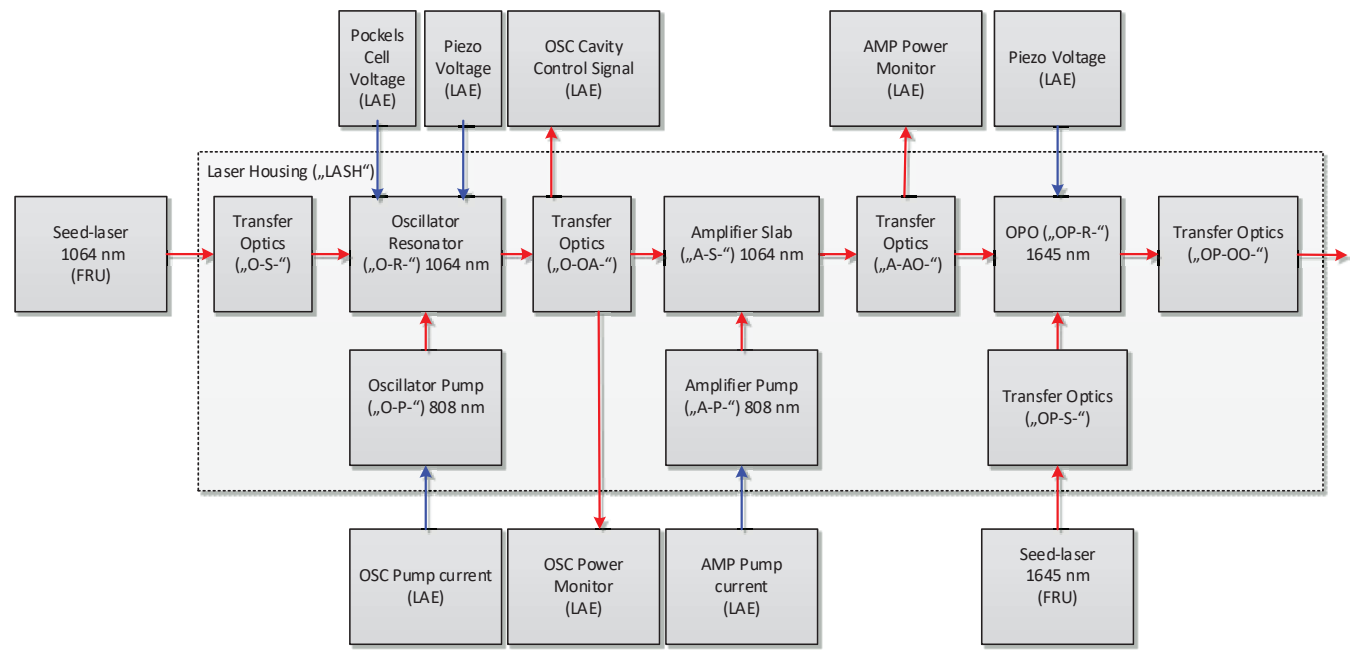

Figure 2. Detailed functional block diagram of optical and electrical functions in LASO. 
The housing includes all feedthroughs that are necessary to deliver the mentioned inputs and outputs.

\subsection{Major design requirements}

For the IPDA measurements the laser output beam of the LASO has to meet several optical requirements. The basic ones are listed in Table 1.

\begin{tabular}{|l|l|}
\hline Parameter & Required output \\
\hline Pulse energy & $>9 \mathrm{~mJ}$ \\
\hline Repetition rate & $20 \mathrm{~Hz}$ double pulse $=40 \mathrm{~Hz}$ single pulse \\
\hline Output wavelengths & $\begin{array}{l}\text { Online: } 1645.5516 \mathrm{~nm} \\
\text { Offline: } 1645.8183 \mathrm{~nm}<\lambda<1645.8326 \mathrm{~nm}\end{array}$ \\
\hline Pulse width & $>10 \mathrm{~ns}$ \\
\hline Spectral purity & $>99.95 \%$ \\
\hline
\end{tabular}

Table 1. Basic optical requirements for LASO.

Besides those basic but still demanding requirements, also the stability of the output beam has to fulfill a number of specifications concerning e.g. the pointing of the beam on different time scales. The main requirements are listed in Table 2. All those requirements include possible systematic deviations between online and offline pulses.

\begin{tabular}{|l|l|}
\hline Parameter & Maximum variation \\
\hline Beam pointing stability & $<750 \mu \mathrm{rad}$ \\
\hline Beam divergence & $<11 \%$ \\
\hline Systematic beam pointing difference between online/offline pulse & $<40 \mu \mathrm{rad}$ \\
\hline
\end{tabular}

Table 2. Optical stability requirements for LASO.

Besides those optical requirements that are derived from the measurement principle and the telescope optics, several constraints have an impact on the optomechanical and thus optical design of the laser: One major design constraint for the MERLIN instrument is the passive thermal control that leads to a large operational temperature range between $15^{\circ} \mathrm{C}$ and $28^{\circ} \mathrm{C}$. At the same time the design has to be compact and lightweight due to the overall restrictions for the whole instrument. The laser has to withstand demanding mechanical loads such as shock up to $270 \mathrm{~g}$ and random vibrations of up to $19.6 \mathrm{~g}(\mathrm{rms})$ in the range $20 \mathrm{~Hz}$ to $2 \mathrm{kHz}$. Additionally, pressure and temperature changes as well as gravitational force change the interface loads that are applied on the baseplate by the housing. LASH.

To meet the lifetime requirement (mission duration $>3.25$ years), avoiding of Laser-Induced Contamination (LIC) is of crucial importance inside the sealed and pressurized laser housing. For that reason, optical mounting is achieved by soldering and also the electrical harness is built up using soldering technology only. In doing so, any outgassing caused by cable shielding or glue residues is avoided entirely. 


\section{OPTICAL DESIGN}

\subsection{Oscillator}

The master oscillator is on one side of the LASO baseplate. The functional design is shown in Figure 3 with color coding for different sections. The active medium is Nd:YAG and as in the FULAS laser the oscillator crystal is pumped from both sides with diodes emitting at $808 \mathrm{~nm}$ that are mounted in two fiber-coupled pump modules [4]. The pump section is marked in purple.

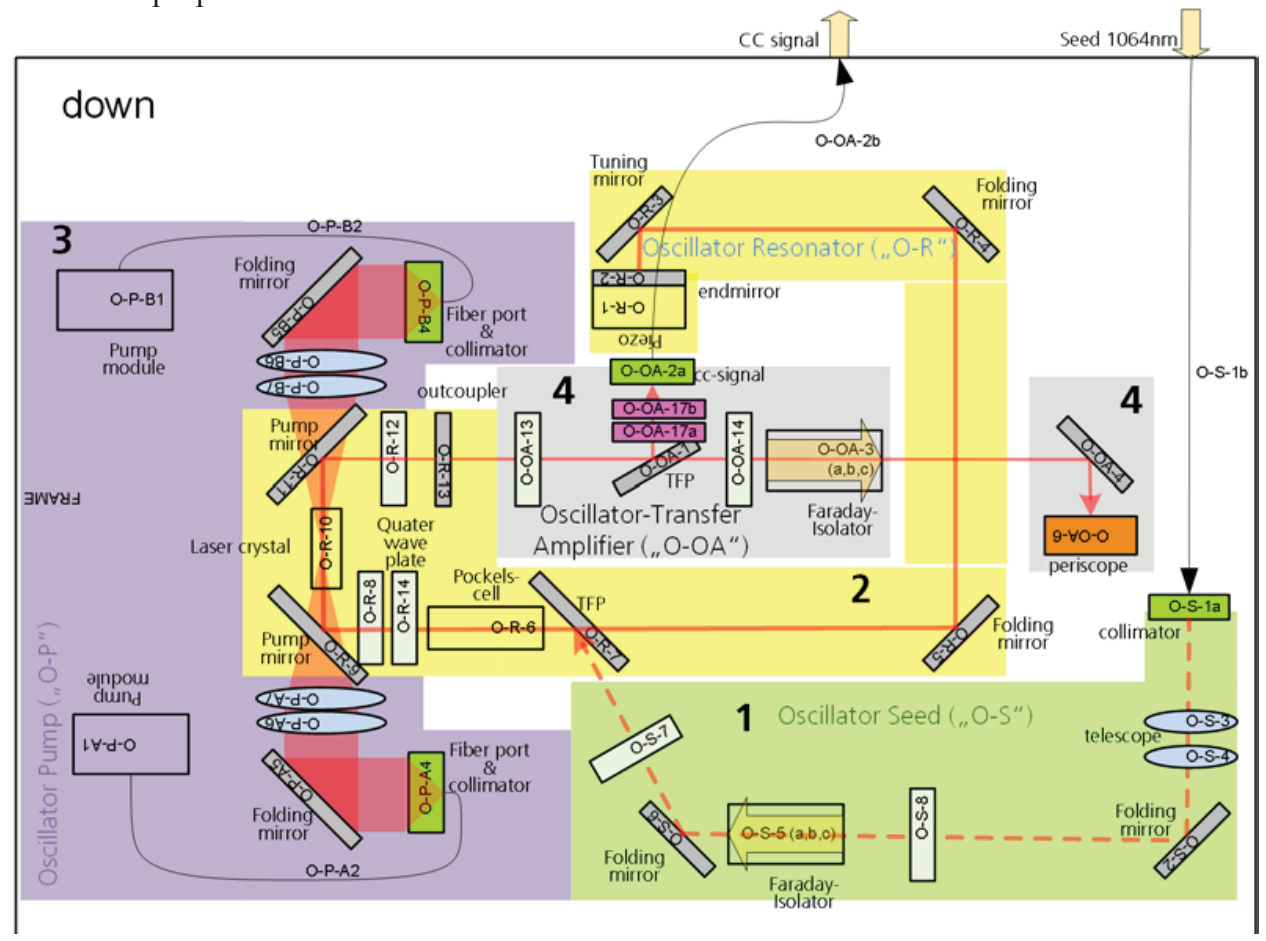

Figure 3. Block diagram of the oscillator side of LASO

To reach the high spectral purity requirements for the OPO signal beam it is necessary to reach very good spectral properties of the OPO pump and therefore with the oscillator output beam. Especially the spectral purity and thus the side mode suppression is crucial. Those requirements are reached with the help of a cavity dither control of the resonator length. For this cavity control an injection seed laser at the laser wavelength is used (green section) that is provided by the FRU. The beam is coupled into the resonator (yellow section) via a thin film polarizer and the resonator length is being varied with the help of a piezo element on one end mirror to determine the optimal position of the mirror. For the pulse emission the piezo is set to that position and the q-switch is activated, that is implemented with a Pockels cell made of a BBO crystal.

The beam is coupled out via a partially reflective mirror and the generated pulses have a pulse energy of $>4.8 \mathrm{~mJ}$ and a duration of $14.5 \mathrm{~ns}$. As in the beam path of the seed laser a Faraday isolator is implemented behind the oscillator to avoid disturbances by back reflections that could lead to a decrease of the spectral properties and in the worst case to the destruction of the oscillator.

\subsection{Amplifier}

The amplifier is positioned on the other side of the baseplate together with the OPO. A periscope is used to guide the beam from the oscillator side to the amplifier side. The block diagram of the second side of the baseplate is shown in Figure 4. 


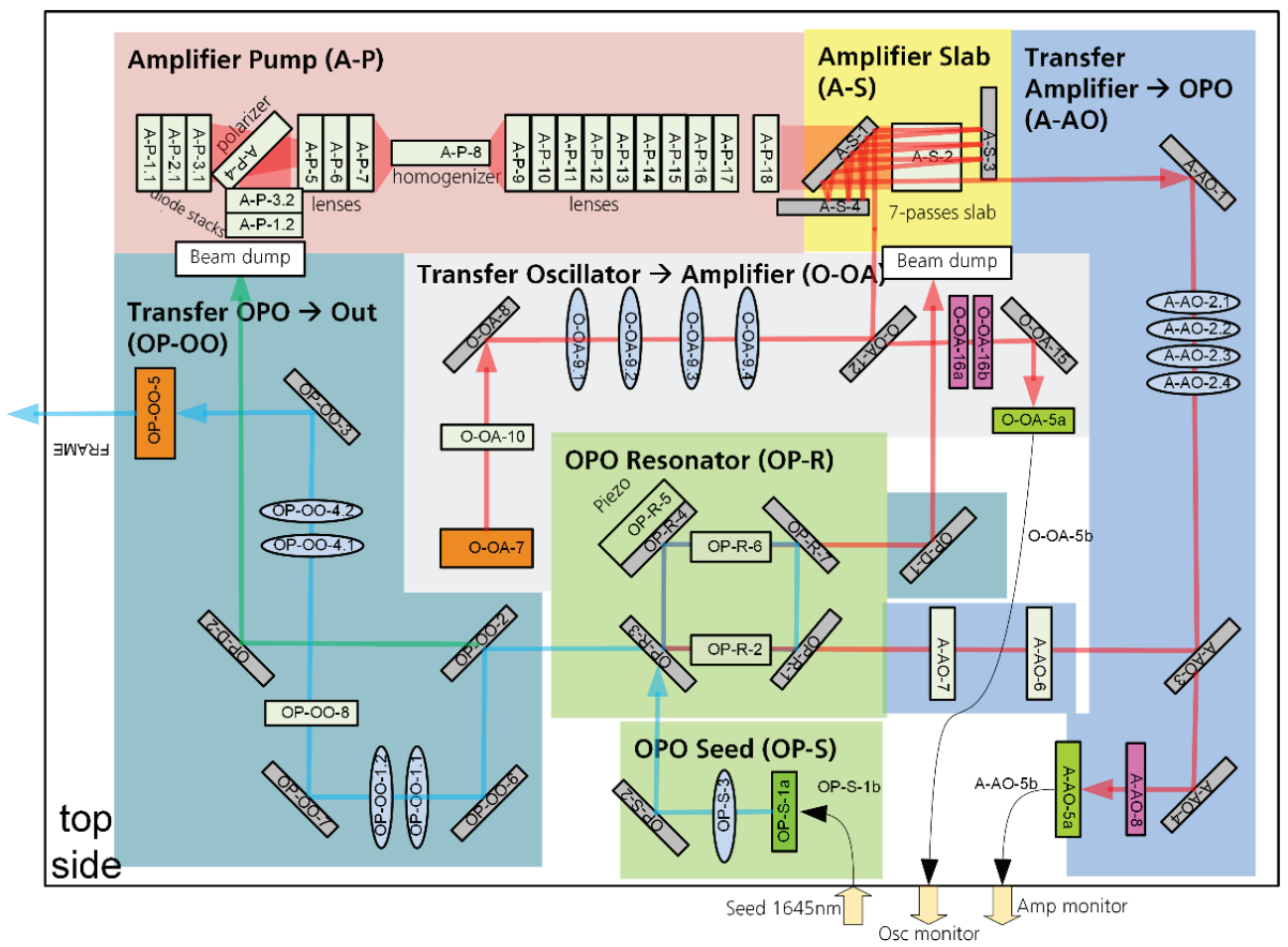

Figure 4. Block diagram of the amplifier/OPO side of LASO

For the amplifier, the InnoSlab design is applied. This concept is ideal for high energy and high efficiency single mode power amplifiers. The Nd:YAG crystal is pumped from one side with high-power diode stacks and several additional beam forming optics to optimize the pump geometry in the crystal (pink section). The limited space for the pump optics is especially challenging and had a strong impact not only on the optics design but also on the required optical mounting system.

The beam coming from the oscillator is first shaped with a cylindrical telescope and then folded seven times through the slab-crystal (concept see Figure 5).

By choosing the correct beam shaping and slab mirror design the beam is widened with every pass in slow axis so that the efficiency is optimized and the fluence kept constant and below the damage threshold.

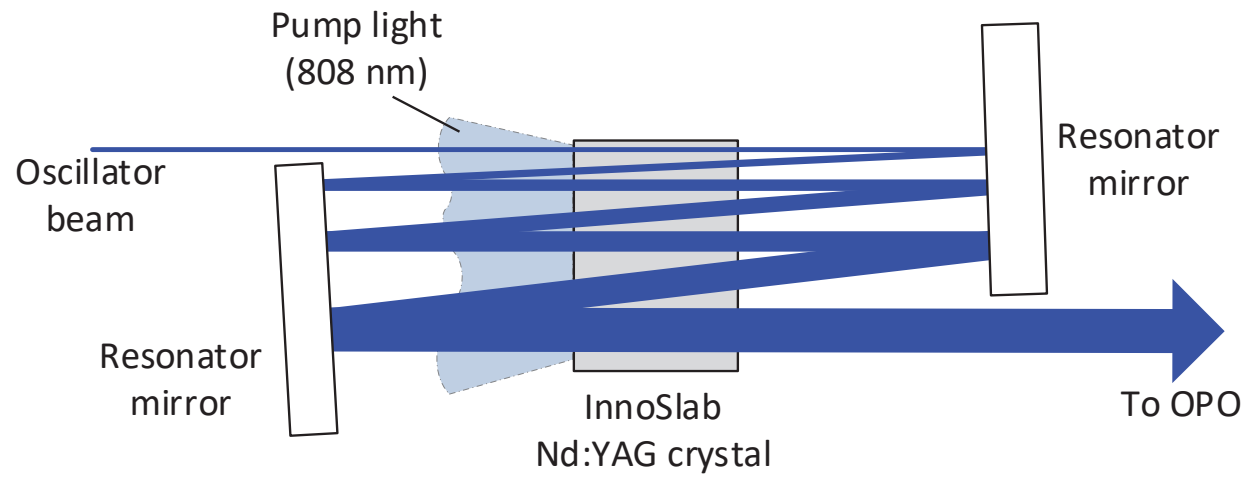

Figure 5. Schematic of folded signal beam path in InnoSlab crystal 
With the input signal of $4.8 \mathrm{~mJ}$ coming from the oscillator the amplifier can generate an output in the range of $30 \mathrm{~mJ}$ to $35 \mathrm{~mJ}$, depending on the exact input requirements of the OPO. The main advantages of this amplifier design are a comparably high efficiency of around $25 \%$ and thus less heat loads, and no thermal lensing in the critical plane of the OPO. Following the amplifier an additional telescope and waveplate are used to shape the beam to the requirements of the OPO.

\subsection{OPO}

For the frequency conversion to the absorption line of methane an OPO is implemented. It consists of two KTP crystals in a ring resonator (green section in Figure 4). The design of the OPO is based on a concept by DLR IPA [6] and has been optimized with the help of a simulation model developed by ILT to increase the efficiency, fit the optical parameters and the mechanical boundary conditions.

The frequency conversion is performed in the X-Z-plane of the crystals so that pump (1064 nm) and signal (1645 nm) have a polarization parallel to the laser bench and the idler $(3010 \mathrm{~nm})$ polarization is perpendicular to that plane. As the spectral properties of the signal beam are very important, the phase mismatch has to be minimized for both online and offline wavelength. To allow this precise phase matching despite the large operational temperature range, both crystal mounts have a temperature control.

One other important parameter is the systematic pointing difference between pulses of the two signal wavelengths, as this has a direct impact on the measurement precision. The initial optical design showed a pointing difference in the range of $\sim 350 \mu \mathrm{rad}$, which was too large as a value $<40 \mu \mathrm{rad}$ is required. With the help of the simulation model, that includes all geometric beam properties, the design could be further optimized by choosing adapted crystal lengths for the two KTP crystals. With that design the systematic pointing difference can be reduced to $0 \mu \mathrm{rad}$ although the alignment tolerances remain.

To fulfill the spectral purity requirements, the OPO resonator employs a mirror on a piezo element for cavity control. Due to the low finesse of the resonator, the possible cavity control methods are limited. In MERLIN the resonator length is adjusted from one online-pulse to the next, by measuring the difference between the wavelength of the injection seeder and the wavelength of the online-pulse. This comparison is performed by the FRU that provides this information to the LAE to set the piezo position. Compared to the online wavelength the absolute wavelength for the offline pulse is not as crucial for the LIDAR measurement. Therefore, for the offline pulse the wavelength of the injection seeder is tuned to match a resonance of the OPO resonator instead of changing the piezo position.

The unused radiation at the idler wavelength and the not converted pump pulse energy are coupled out of the OPO cavity at two respective one dichroic mirrors. The beams are then guided to two beam dumps, based on volume absorber materials for the respective wavelengths. The beam dumps are thermally coupled to the thermal harness. The extracted signal beam is shaped with another telescope to match the stringent requirements on the beam divergence in both directions.

\subsection{Bread Board Setup}

The performance of the optical design was tested on a breadboard setup. The optical design is representative with respect to the flight design and also the interfaces to LAE and FRU are the same. The optics are mounted in conventional mounts, so that more parameters can be monitored, and the system is more flexible compared to the soldered flight design. The disadvantage is, that the breadboard is not representative concerning the mechanical and thermal properties and stabilities. 


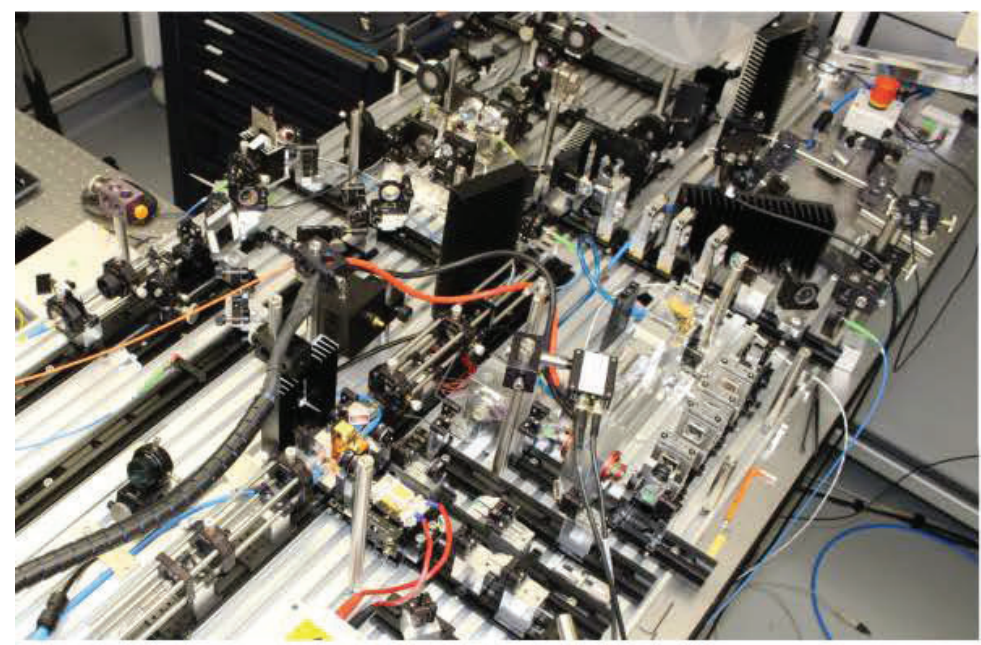

Figure 6. Photo of the MERLIN LASO Breadboard setup.

This setup was used to verify that all optical requirements that do not mainly depend on those stabilities can be met with using the LAE and FRU as controls. All requirements listed in Table 1 were successfully verified, which includes the important spectral purity requirement.

Due to the instability of the system it was difficult to measure the pointing stabilities which includes the low systematic pointing difference between online and offline pulse. But it could be shown that within the general pointing instability of the breadboard, no difference between the pointing of the two signals beams could be found with the breadboard.

\section{OPTOMECHANICAL MOUNTS}

The optomechanical mounts must meet the following requirements to ensure safe and stable long-term operation of the laser system:

- low tilting during environmental loads

- no component outgassing

- multiple adjustment during assembly (re-melting of the solder joint)

- compact design

- compensation of the different thermal expansion coefficients between base plate and optical components

- interchangeability of the mounts during assembly

The basic types of mounts that are used in MERLIN and their design features are described below. All mounts including their variations have been developed in the frame of the OPTOMECH projects and, except the KTP mount, were successfully pre-tested within the FULAS project [4],[5].

\subsection{Composite-Mount}

The composite mount (C-mount) is most often used within the MERLIN LASO. The presented C-mount is the basic design for optical mounts. Several other optical mounts were developed e.g. for periscope optics or spherical lenses and are based on the same design. The recurrent task for all optomechanical mounts is to join optical materials such as quartz, BK7 or SF6 to a laser base plate made of aluminum. The physical properties, especially the thermal expansion coefficients of these materials, are very different and have to be compensated to reach the high stabilities. This compensation is accomplished by an optimized composition of materials in-between the optical component and the laser base plate as well as by design elements. A picture of a basic C-mount is shown in Figure 7. 


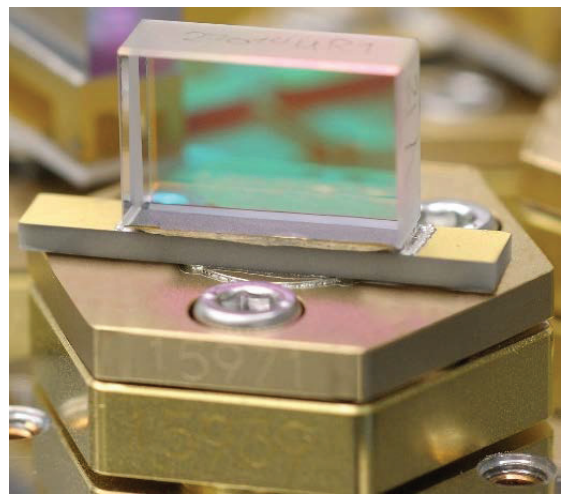

Figure 7. Photo of a basic C-Mount.

The C-mount was realized by means of Fraunhofer ILT's Pick \& Align resistance soldering technique. A crucial element in this process is the active alignment of optical components in the solder pool. A six-axis manipulator enables the components to be positioned with extreme precision during the soldering process. To further increase the strength and reproducibility of the joint produced, the soldering technique was investigated in terms of long-term stability and positional accuracy. In particular, surface properties and metallization of the joining partners decisively influence the joint and must be tailored to thermal and mechanical stresses. In addition, various solder geometries were investigated in order to optimize the positional accuracy of optical components both during the soldering process and once exposed to the environment.

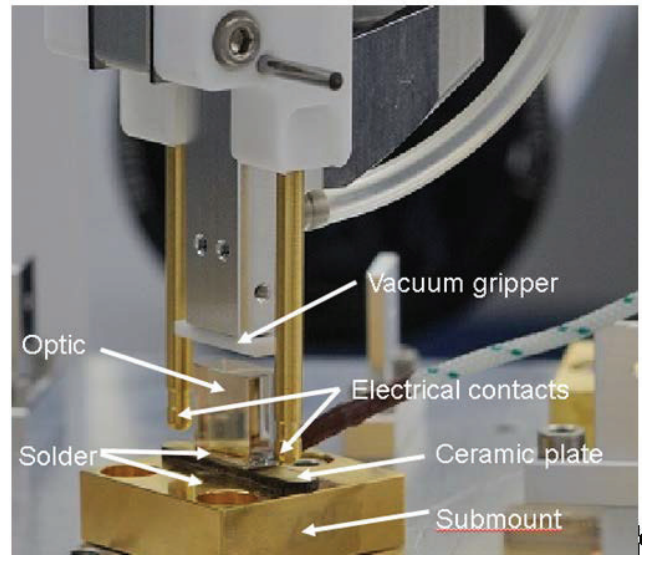

Figure 8. Adjustable Pick\&Align mirror on a submount and details of the mounting tool

The most critical stability requirements within the MERLIN LASO regarding position accuracy between optics and laser base plate have to be fulfilled in the oscillator and the OPO. The deviations from the alignment position shall be smaller than $\pm 10 \mu \mathrm{rad}$ after non-operational thermal loads and during operational thermal and mechanical loads. Measurements show that the C-mount exhibits an alignment stability of the optical component up to the possible accuracy of measurement for the arrangement in the climate chamber while thermal cycling. An exemplary test result for a C-Mount exposed to temperatures from $-30^{\circ} \mathrm{C}$ to $50^{\circ} \mathrm{C}$ is shown in Figure 8 . 


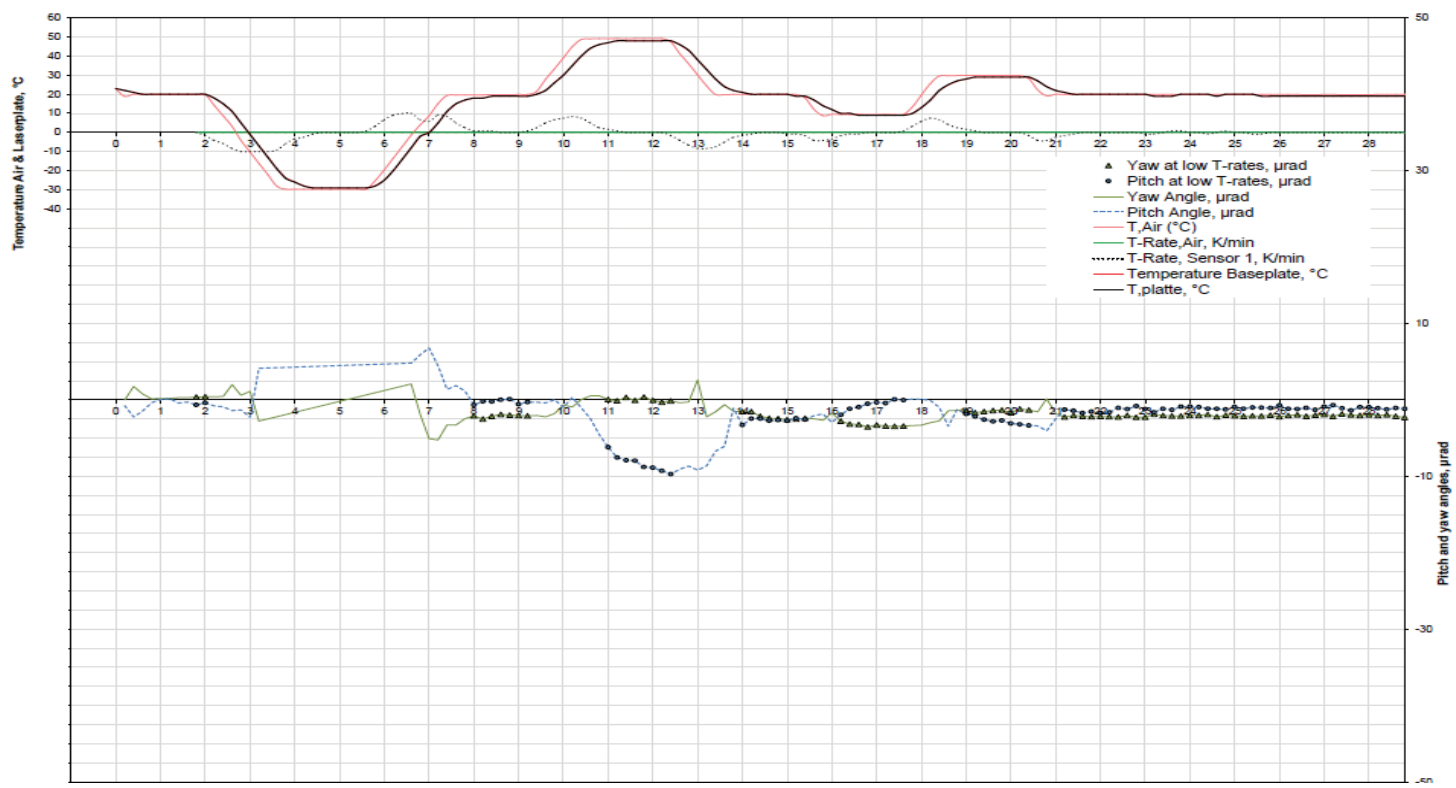

Figure 9. Exemplary thermal cycle test with a Composite-Mount; pitch angle (blue), yaw angle (green) dependent on temperature

\subsection{Faraday Isolator}

The Faraday isolator includes a magnetic field assembly and two optical inserts with a TGG crystal (see Figure 9). The magnets inside the housing are encapsulated by a tube around the free aperture and the housing provides fittings between the individual sections to avoid particle transmission.

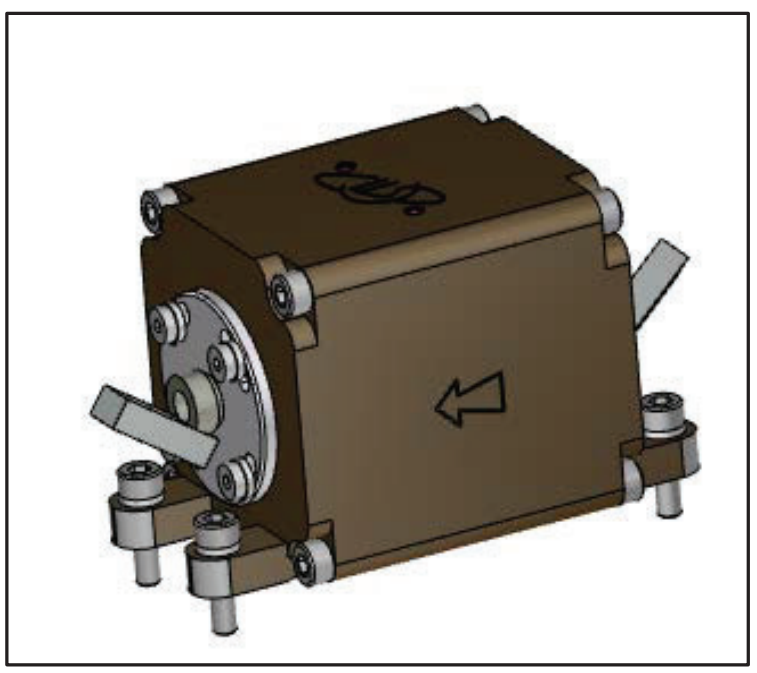

Figure 10. CAD Model of a Faraday Isolator.

The optical inserts are designed in accordance with the same design principles as for the C-Mount: A suitable material pairing reduces the mechanical stress in the optical components due to CTE mismatch. All parts of the insert are soldered so that all components are fixed without glue. Both inserts can be rotated to align the Faraday isolator. After the alignment is finished, the inserts can be fixed on the housing of the magnetic field assembly by bolts. 


\subsection{Pockels Cell}

In addition to the mounting of the BBO crystal, the high electrical voltage represents a challenge for the design of the Pockels cell. To prevent any flashover occurrence, design criteria like geometry, metallization, material selection and manufacturing processes were considered. The resulting Pockels cell design consists of a metallized BBO crystal, two metallized electrical contacts and four metallized housing parts made of ceramic. The electrical contacts additionally perform the function of the mechanical mount of the BBO crystal.

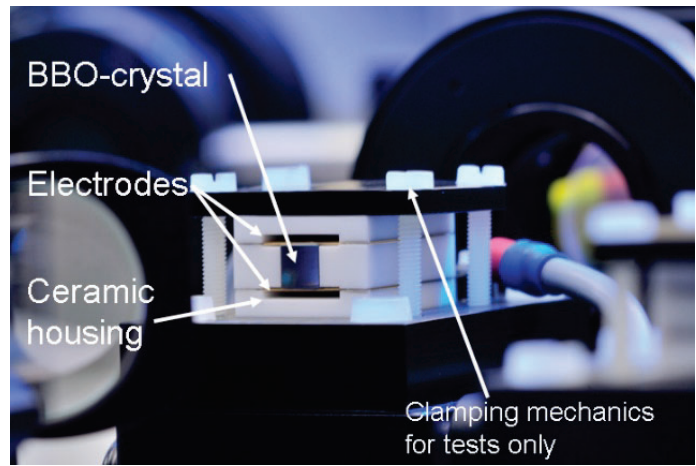

Figure 11. Picture of the Pockels cell.

This assembly (Figure 10) is joined to an optomechanical mount similar to the C-mount by the Pick\&Align soldering process. Besides the mechanical interface to the laser plate, the Pockels cell mount features electrical transfer interfaces to the harness of the laser system.

\subsection{KTP Crystal Mount}

The KTP crystal mount is used in the OPO, in which two KTP crystal-mounts with different crystal lengths are included (see section 3.3). The KTP crystal mount consists of a KTP oven and an optomechanical sub-mount as basis. The submount acts as a mechanical holder and also provides an electrical transfer interface for the thermal control. Each KTP oven consists of two heater bars and a KTP crystal soldered between them (see Figure 11). Each heater bar has a heater plate integrated by a soldering technique. In addition, the upper heater plate features a temperature sensor for thermal control. The design of the heater bar contributes to a homogeneous heat distribution within the KTP crystal due to reduced contact area to the mounting plate. For mounting or gripping purposes each heater bar contains a mounting plate.

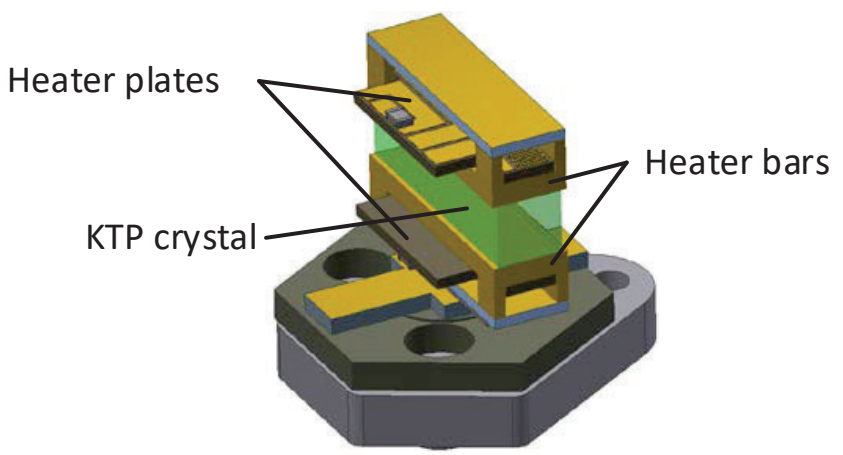

Figure 12. KTP crystal-Mount (without electrical contacts) 


\section{ANALYSES}

There are two mechanisms that lead to a misalignment of the laser beam: The variation of mechanical loads at the interfaces to LASH and the change of the LASO baseplate temperature.

\subsection{Interface Loads LASH/LASO}

The interface loads change due to the change of the difference between inner and outer pressure of the housing, the orientation and magnitude of the gravity vector and temperature changes with respect to the integration temperature. All those changes appear on different time scales as on long-term scale for ground-to-orbit effects or on mid-term scales for one orbit or one month.

A mechanical analysis was performed to calculate the effect those interface loads have on the baseplate at the position of each optical mount. Based on the results the tilting of each mount was derived for each possible combination of interface loads.

\subsection{C-Mount thermomechanical stability}

To analyze the effect of the baseplate temperature variation on the alignment, results from the OPTOMECH project were used. Prior to its integration each mount was tested in the climate chamber and the tilting was observed before and after a non-operational cycle and during an operational cycle. Compared to MERLIN the temperature ranges were a bit larger with a non-operational range of $-30{ }^{\circ} \mathrm{C}$ to $50{ }^{\circ} \mathrm{C}$ and an operational range of $10{ }^{\circ} \mathrm{C}$ and $30{ }^{\circ} \mathrm{C}$, so the data can be seen as worst case. The example of a measurement result is shown in Figure 9.

Overall 35 mounts were tested and based on previous analyses only those with a tilting of $<10 \mu$ rad after the nonoperational cycle and a tilting of less than $1 \mu \mathrm{rad} / \mathrm{K}$ over the whole operational temperature range were selected. Of the 35 mounts 26 fulfilled those requirements which corresponds to $74 \%$. The other mounts can be used for less alignment sensitive optics. The standard deviations at different temperature ranges for the selected mounts were calculated as listed in Table 3.

Table 3. Standard deviations of C-mount normal distributed tilt statistics after non-operational and during operational cycle

\begin{tabular}{|c|c|c|c|c|c|}
\hline \multirow{2}{*}{} & \multirow{2}{*}{ mount } & \multicolumn{2}{|c|}{$\mu \mathrm{rad}$} & \multicolumn{3}{c|}{$\mu \mathrm{rad} / \mathrm{K}$} \\
\cline { 3 - 6 } & & \multicolumn{2}{|c|}{ non-op } & \multicolumn{2}{|c|}{ op } \\
\hline total quantity & 35 & yaw & pitch & yaw & pitch \\
\hline nonop $<10 \mu \mathrm{rad} \&$ op $<1 \mu \mathrm{rad} / \mathrm{K}$ & 26 & 1.3 & 3.0 & 0.21 & 0.34 \\
\hline
\end{tabular}

\subsection{Analysis of Oscillator and Amplifier}

To obtain the parameters of the $1064 \mathrm{~nm}$-beam at the output of the amplifier and thus the OPO input, a ray transfer matrix analysis was used for the oscillator and amplifier. The impact of the interface loads can easily be calculated as the tilting of each optic is specified and can directly be incorporated in the model.

As the temperature changes of the LASO baseplate lead to a statistically distributed tilting of the optics, a Monte Carlo analysis was done: For each of 10000 model runs each mount was randomly tilted, the random figure was weighted with a normal distribution with the standard deviations taken from Table 3. This was done for different time scales.

\subsection{Analysis of OPO}

The analysis of the OPO is more complex due to the frequency conversion as non-linear process. For the calculation of the OPO output parameters the same numerical OPO model is used as for the optimization of the OPO design (see section 3.3). The impact of the interface loads is calculated by changing the tilting of each optic as calculated in the mechanical analysis.

The disadvantage of this split-step model is the relatively high calculation duration of two minutes per model run. Together with the large number of critical input parameters, the total duration of a Monte Carlo Analysis for the thermal analysis would be too long. This is why a worst-case calculation is performed with the $99,7 \%$ probability for each output parameter as result. As for the oscillator and amplifier a calculation is performed for each time scale. 


\subsection{Results of the performance analysis}

To compare the analysis results with the requirements, the different effects have to be combined. The operational and non-operational thermal loads are assumed to be statistically independent effects. The deviations in output parameters caused by them are added geometrically. The effect of interface loads is considered as a constant shift of each optic and is added linearly to the thermal loads.

For both mid-term time scales (one orbit and one month) the calculations show compliance to the requirements on all calculated output requirements. No short-term changes are expected.

The major results for the long-term scale are listed in Table 4.

Table 4. Results of optical performance analysis for long term time scale for online / offline wavelength

\begin{tabular}{|l|l|l|l|l|l|}
\hline Parameter & Interface loads & $\begin{array}{l}\text { After non- } \\
\text { operational } \\
\text { temperature } \\
\text { cycle }\end{array}$ & $\begin{array}{l}\text { Thermal } \\
\text { long-term } \\
\text { variation } \\
\pm 6.5 \mathrm{~K}\end{array}$ & Total & Requirement \\
\hline Beam pointing stability / $\mu \mathrm{rad}$ & $31 / 30$ & $347 / 353$ & $257 / 262$ & $463 / 470$ & $<750$ \\
\hline Beam divergence horizontal / \% & $0.6 / 0.5$ & $3.1 / 3.6$ & $3.3 / 3.7$ & $5.1 / 5.7$ & $<11$ \\
\hline $\begin{array}{l}\text { Systematic beam pointing } \\
\text { difference between } \\
\text { online/offline pulse / } \mu \mathrm{rad}\end{array}$ & 4 & 10 & 11 & 21 & $<40$ \\
\hline
\end{tabular}

All parameters for the long-term scale are well within the requirements although the inputs and calculations are considered worst case.

\section{CONCLUSION}

In this paper we presented the optical design of the MERLIN laser and the optomechanical design of the basic mounts that are used within the laser. Additionally, we explained the analyses, that were executed to show the performance of the laser with those mounts under the defined environmental loads. The results show that the requirements on the output beam will be fulfilled.

The CDR of the MERLIN LASO was completed successfully in September 2020 including the presented designs and analyses.

\section{ACKNOWLEDGEMENTS}

The R\&D project underlying the report, »MERLIN Phase $\mathrm{C} / \mathrm{D} «$, has been carried out on behalf of the Federal Ministry for Economic Affairs and Energy BMWi under the grant number 50EP1601. The work in »MERLIN« is being carried out on behalf of the DLR Space Administration, subcontracted by Airbus Defence and Space GmbH in phases C/D.

The authors would like to thank the whole MERLIN project team at DLR, CNES, Airbus DS, SpaceTech GmbH, von Hoerner \& Sulger GmbH and Fraunhofer ILT, for their continuous support and their fruitful cooperation in this challenging project. In particular, we would like to thank Dimitrios Kokkinos, Hans Huber, Matthias Winzen, Dominik Esser and Jochen Wüppen. 


\section{REFERENCES}

[1] C. Stephan et al., "MERLIN - a space-based methane monitor", Lidar Remote Sensing for Environmental Monitoring XII, Proc. of SPIE, Vol. 8159, (2011)

[2] P. Flamant, G. Ehret, B. Millet, M. Alpers, "MERLIN: a French-German mission addressing methane monitoring by LIDAR from space", Proc. of 26th ILRC, Porto Heli, Greece, (2012).

[3] Christian Wührer, Christopher Kühl, Stefano Lucarelli, M. Bode, "MERLIN: overview of the design status of the lidar Instrument," Proc. SPIE 11180, International Conference on Space Optics — ICSO 2018, 1118028 (12 July 2019);

[4] Sven Hahn, Markus Bode, Jörg Luttmann, Dieter Hoffmann, „FULAS: High energy laser source for future LIDAR applications" Proceedings of ICSO 2018, Crete, Greece Oct. 2018.

[5] J. Löhring, M. Winzen, H. Faidel, J. Miesner, D. Plum, J. Klein, O. Fitzau, M. Giesberts, W. Brandenburg, A. Seidel, N. Schwanen, D. Riesters, S. Hengesbach, H.-D. Hoffmann, "Key optical components for spaceborne lasers," Proc. SPIE 9730, Components and Packaging for Laser Systems II, 973000 (22 April 2016);

[6] Andreas Fix, Christian Büdenbender, Martin Wirth, Mathieu Quatrevalet, Axel Amediek, Christoph Kiemle, Gerhard Ehret, "Optical parametric oscillators and amplifiers for airborne and spaceborne active remote sensing of CO2 and CH4," Proc. SPIE 8182, Lidar Technologies, Techniques, and Measurements for Atmospheric Remote Sensing VII, 818206 (30 September 2011) 